\title{
Guitar: An R/Bioconductor Package for Gene Annotation Guided Transcriptomic Analysis of RNA-Related Genomic Features
}

\author{
Xiaodong Cui, ${ }^{1}$ Zhen Wei, ${ }^{2,3}$ Lin Zhang, ${ }^{4}$ Hui Liu, ${ }^{4}$ Lei Sun, ${ }^{5}$ Shao-Wu Zhang, \\ Yufei Huang, ${ }^{1}$ and Jia Meng ${ }^{2,3}$ \\ ${ }^{1}$ Department of Electrical and Computer Engineering, University of Texas at San Antonio, San Antonio, TX 78230, USA \\ ${ }^{2}$ Department of Biological Sciences, HRINU, SUERI, Xian Jiaotong-Liverpool University, Suzhou 215123, China \\ ${ }^{3}$ Institute of Integrative Biology, University of Liverpool, Liverpool L69 3BX, UK \\ ${ }^{4}$ School of Information and Electrical Engineering, China University of Mining and Technology, Xuzhou 221116, China \\ ${ }^{5}$ School of Information Engineering, Yangzhou University, Yangzhou, Jiangsu 225127, China \\ ${ }^{6}$ School of Automation, Northwestern Polytechnical University, Xian, Shaanxi 710027, China
}

Correspondence should be addressed to Jia Meng; jia.meng@xjtlu.edu.cn

Received 10 February 2016; Revised 5 April 2016; Accepted 11 April 2016

Academic Editor: Ivan Merelli

Copyright (C) 2016 Xiaodong Cui et al. This is an open access article distributed under the Creative Commons Attribution License, which permits unrestricted use, distribution, and reproduction in any medium, provided the original work is properly cited.

Biological features, such as genes and transcription factor binding sites, are often denoted with genome-based coordinates as the genomic features. While genome-based representation is usually very effective in correlating various biological features, it can be tedious to examine the relationship between RNA-related genomic features and the landmarks of RNA transcripts with existing tools due to the difficulty in the conversion between genome-based coordinates and RNA-based coordinates. We developed here an open source Guitar R/Bioconductor package for sketching the transcriptomic view of RNA-related biological features represented by genome based coordinates. Internally, Guitar package extracts the standardized RNA coordinates with respect to the landmarks of RNA transcripts, with which hundreds of millions of RNA-related genomic features can then be efficiently analyzed within minutes. We demonstrated the usage of Guitar package in analyzing posttranscriptional RNA modifications (5-methylcytosine and N6-methyladenosine) derived from high-throughput sequencing approaches (MeRIP-Seq and RNA BS-Seq) and show that RNA 5 -methylcytosine $\left(\mathrm{m}^{5} \mathrm{C}\right)$ is enriched in $5^{\prime} \mathrm{UTR}$. The newly developed Guitar R/Bioconductor package achieves stable performance on the data tested and revealed novel biological insights. It will effectively facilitate the analysis of RNA methylation data and other RNA-related biological features in the future.

\section{Introduction}

Genome-based coordinates, which consist of the name of chromosome and the starting/ending coordinates, have been widely used to denote the genomic location of various biological features, such as genes, SNPs, and transcription factor binding sites (TFBS). With genome-based coordinates, the relationship between different biological features can be easily inferred. Currently, genomic features (biological features represented by genome-based coordinates) have become the basis of many bioinformatics tools in various biological data processing pipelines, and dedicated types of operation are also available [1]. While genome-based coordinates are very useful for analysis of genome related biological features, it can still be tedious for analysis or visualization of RNA-related features, such as RNA N6-methyladenosine $\left(\mathrm{m}^{6} \mathrm{~A}\right)$ and RNA 5-methylcytosine $\left(\mathrm{m}^{5} \mathrm{C}\right)[2]$.

As an emerging layer of gene expression regulation, posttranscriptional RNA modifications, including $\mathrm{m}^{6} \mathrm{~A}$ and $\mathrm{m}^{5} \mathrm{C}$, are recently found to play various important roles in a number of biological processes, such as translation efficiency [3], microRNA processing [4], RNA-protein interaction [5], RNA stability [6], and pluripotency [7]. Together with the development of new sequencing approaches [8-11] for unbiased profiling of the posttranscriptional RNA modifications, a number of bioinformatics tools [12, 13] have been created 
for interpretation of these datasets. A mammalian RNA methylation database [14] has been created that paved the way for a systematic understanding of the RNA methylome regulation mechanism [15]; however, to our knowledge, no bioinformatics effort has been made specifically for effective visualization of RNA methylation features from global level. Conceivably, the functions of RNA-related features are likely to be related to the landmarks of RNA transcripts, that is, transcription starting site (TSS), start codon, stop codon, and transcription ending site (TES), and the existing tools developed for genome-based features are not effective for analysis of RNA methylation data.

Compared with genome-regulated biological features (e.g., histone modifications and TFBS), visualization of RNArelated features (such as RNA methylation sites) represented in genomic coordinates is nontrivial due to the following reasons:

(i) Increased Ambiguity due to Isoforms. The ambiguity in genome-based coordinates is often due to the repeats. Compared with genome, transcriptome has increased complexity due to transcript isoform and alternative splicing. It is possible that an RNA methylation site falls into the CDS of one transcript but on the $3^{\prime}$ UTR of another isoform transcript. It is known that many genes have very large number of isoform transcripts, so it may be impossible to specifically assign a genomic feature aligned on this gene to a particular isoform transcript.

(ii) Variation in Transcript Length. In human and mouse, transcripts vary greatly in size. It is important to notice that 1000 base pairs (bp) from start codon may be a long distance for shorter genes but not for the longer ones. For genomic coordinates, bp has been shown to be a reasonable unit; however, when measuring distance between two RNA-related features, a relative unit standardized by the entire width of the transcript may be more suitable. This idea has been used widely today in many studies. Please note that the technical resolution remains the same when measured in bp on shorter or longer transcripts. When a standardized coordinate is used, longer transcripts actually obtain a higher resolution in terms of standardized coordinate compared with the shorter transcripts. Compared with a transcript with $20 \mathrm{k} \mathrm{bp}$, it is more difficult to tell whether the RNA methylation site on a 200 bp transcript is close to its stop codon, even though the technical resolution remains the same.

(iii) Complexity in Landmarks of RNA Transcript. For histone modifications and transcription factor binding sites, landmarks of interests are often the transcription starting site (TSS) and transcription ending sites (TES). For RNA methylation, two additional landmarks are the start codon and stop codon of mRNA and the setting is further complicated by the existence of long noncoding RNA (lncRNA) and various small RNA families. Furthermore, some mRNAs may not have $3^{\prime}$ UTR or $5^{\prime}$ UTR. Additionally, the $5^{\prime} \mathrm{UTR}$, CDS, and $3^{\prime}$ UTR are apparently of different length for most genes, so the 3 components need to be standardized independently when summarized or compared with other transcripts. There are already tools, such as ngs.plot [16], developed to handle TSS and TES, but to our knowledge, none supports more detailed structure (start codon and stop codon). It is important to include all RNA landmarks and discriminate coding and noncoding RNAs when analyzing RNA-related features due to their intrinsic property.

For the aforementioned reasons, a dedicated approach needs to be developed for visualization of RNA methylation data and other RNA-related biological features.

\section{Design and Implementation}

We develop here an open source R package Guitar for gene annotation guided transcriptomic analysis of RNA-related genomic features, such as RNA methylation sites denoted in genome-based coordinates. The approach is detailed next.

2.1. Guitar Coordinates. To visualize the multiple RNArelated features together, transcripts of different length need to be standardized in the first place. For this purpose, we constructed the Guitar coordinates, which is essentially the genomic projection of the standardized transcriptomic coordinates. Specifically, each component of a single transcript is divided into a number of bins of equal width. For long noncoding RNA, the whole transcript is a single component; for mRNA, there are 3 components, that is, $5^{\prime} \mathrm{UTR}$, CDS, and $3^{\prime}$ UTR. Their genomic projected coordinates are then obtained with the help of GenomicFeatures R/Bioconductor package [1]. Please note that of interest are the mature mRNA and IncRNA, and it is possible that a specific bin may span introns. The generated Guitar coordinates are essentially still genome-based coordinates but clearly associated with landmarks of transcript, for example, 0.2 standardized lncRNA length from the TSS. The procedures for generating Guitar coordinates are illustrated in Figure 1.

2.2. Guitar Coordinates of a Transcriptome. As mentioned previously, for mRNA, of interest are usually 3 components rather than a single one, that is, $5^{\prime} \mathrm{UTR}$, CDS, and $3^{\prime} \mathrm{UTR}$. Consistently, the Guitar coordinates need to be generated separately for all the 3 regions. In order to make the 3 components comparable, each component is standardized independently and contributes to $1 / 3$ of the entire coding transcript (the difference between $5^{\prime} \mathrm{UTR}$, CDS, and $3^{\prime} \mathrm{UTR}$ in size can also be reflected in the analysis by Guitar package). For lncRNA, this is not needed and the Guitar coordinates are generated for the entire lncRNA.

Due to the existence of isoform ambiguity, the same genomic location may be associated with multiple transcripts and thus related to multiple Guitar coordinates. To ensure the specificity of the generated Guitar coordinates, filtering of highly ambiguous transcripts may be needed. Two filters are implemented. Firstly, a length filter is implemented to select transcripts longer than a user-defined threshold. This 


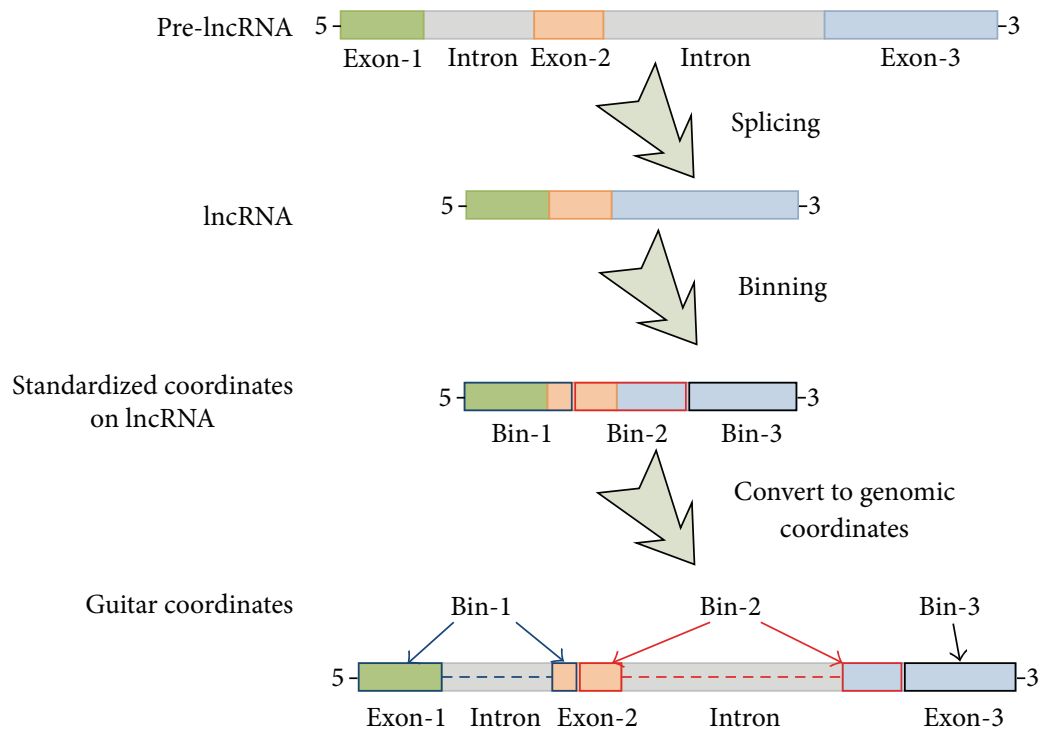

FIgURE 1: Guitar coordinates. This figure illustrates how the Guitar coordinates are generated based on 3 bins on lncRNA transcript. The bins may be split into multiple sections of the transcript represented by GRangesList object, which can be conveniently compared with other genome-based features to reflect their distribution. In practice, if $70 \%$ of a feature overlap $5^{\prime} \mathrm{UTR}$ and $30 \%$ CDS, then it is likely that, the feature overlaps with more Guitar Coordinates (GCs) composed from $5^{\prime}$ UTR than from CDS. As a result, more GCs from $5^{\prime}$ UTR will report the overlapping of this feature, and the resulting weight will precisely reflect the $70 \%$ and $30 \%$ division.

is to ensure the generated Guitar coordinates have sufficient resolution from the technology perspective with the data analyzed. For techniques with single-base resolution, this cutoff can be smaller (e.g., $10 \mathrm{bp}$ ), while, for techniques with lower resolution, it should be larger (e.g., $100 \mathrm{bp}$ ). Secondly, an ambiguity filter is implemented to discard genes with too many isoforms, which can cause ambiguity in the feature assignment stage. The implementation of this filter is mainly to reduce memory usage and save computation time (see Supplementary Material Figure S1 and Table S1 for a comparison of different settings in Supplementary Material available online at http://dx.doi.org/10.1155/2016/8367534). In addition, the filter may be necessary for less-studied species with problematic gene annotation. The second filter can filter out a number of genes with higher isoform ambiguity, and doing so will significantly decrease the memory usage for the constructed Guitar coordinates and save computation time. For this purpose, we implemented a simple strategy by counting the number of overlapping transcripts on the genome, that is, checking the number of transcripts sharing exons. In the default setting of Guitar package, we filtered out transcripts that overlap with more than 3 other transcripts on the genome. The parameter provides reasonable good results in the data tested and can be easily customized by the user. After applying the ambiguity filter, if a genomic feature still overlaps with multiple transcripts, the ambiguity will be factored in the analysis. Specifically, if a site is located on both CDS and $3^{\prime}$ UTR of the same transcript, then the Guitar coordinates associated with those components should have overlapped with that genomic feature, and those coordinates are labeled as associated with the genomic feature with weight 1 , indicating the feature is fully associated with this transcript. However, if a genomic feature is associated with $n$ transcripts, that is, it overlaps with $n$ transcripts, then the overlapping coordinates are associated with that genomic feature with weight $1 / n$, reflecting the ambiguity in association.

2.3. Transcriptomic View of Genomic Features. To sketch the transcriptomic view of genomic features, the Guitar coordinates can be compared with other genomic features, and the number of overlapped features can then be counted and standardized on mRNA and lncRNA, respectively. The distribution of genomic features on RNA will then be summarized and visualized by ggplot 2 package for quality graphics [17]. We also provided a convenient function GuitarPlot for fast visualization of various genomic features in different formats. The general working procedures for Guitar package are shown in Figure 2.

Besides the aforementioned structure, there are a few more useful features provided by Guitar package:

(i) Including neighborhood DNA regions for comparison. The neighborhood DNA regions (promoter region and its complementary DNA at the $3^{\prime}$ end) can be optionally included in analysis of Guitar package. This will be useful for analyzing genomic features that are related to both DNA and RNA, such as H3K4me3 ChIP-Seq data.

(ii) Ambiguous Assignment of the Ambiguous Genomic Features. Due to ambiguity of heterogeneous transcriptome, some genomic features overlap with more than one transcript and thus with ambiguous belongings. In the Guitar package, ambiguous features are also counted in the analysis, with their weights equally divided between all the overlapping transcripts. However, we can optionally filter out genomic features 


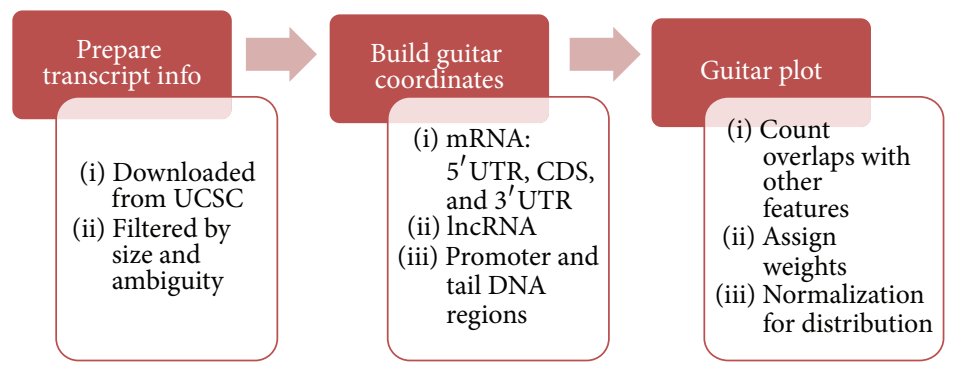

FIGURE 2: Workflow of Guitar package. The gene annotation can be automatically downloaded from Internet or provided as a $T x D b$ object [1] by the user. After filtering transcripts that may not provide useful information, Guitar coordinates of different components are built independently, with which the actual transcriptomic distribution of genomic features can be observed.

overlapping with more than a predefined number of transcripts (Default 5) to completely discard the impact of these highly ambiguous features.

(iii) Resizing the Components in Visualization. In practice, different components of mRNA, that is, $5^{\prime} \mathrm{UTR}$, CDS, and $3^{\prime} \mathrm{UTR}$, are of quite different width. $5^{\prime} \mathrm{UTR}$ is usually much shorter than $3^{\prime}$ UTR and CDS in mouse and human. Although these components are treated independently when building the Guitar coordinates, we still calculated the average length of each component across different genes, so the true relative width can be optionally reflected in the plot generated from Guitar package; however, doing so may make the 5 'UTR region too small for clear observation.

(iv) Connection with ggplot2 for More Complex Graphics. The Guitar may optionally return intermediate results, which can be reused in the ggplot 2 package for more complex graphics. This is designed for the advanced users only and not recommended.

\section{Results}

We developed the Guitar R/Bioconductor package for gene annotation guided transcriptomic analysis of RNA-related genomic features. It is currently and publicly available from Bioconductor. In this section, we show the application of Guitar package to the analysis of RNA methylation sites denoted as genomic features with a few examples. More information is available from the documentation of the Guitar R/Bioconductor package.

3.1. Case Study 1: RNA N6-Methyladenosine from MeRIP-Seq. In the first example, we study MeRIP-Seq data [10] profiling of the transcriptome RNA N6-methyladenosine $\left(\mathrm{m}^{6} \mathrm{~A}\right)[18,19]$ sites in human HepG2 cell lines. The RNA $\mathrm{m}^{6} \mathrm{~A}$ methylation sites are obtained with exomePeak R/Bioconductor package [12]. Compared with other peak calling algorithms, one added flexibility of the exomePeak package is to detect only the highly methylated sites. With a larger "IP/input ratio," exomePeak will report only the highly methylated sites; that is, a higher proportion of a specific kind of RNA molecule carries methylation at this site.
We implemented exomePeak setting different "IP/input ratio" $(1,2,4$, and 8$)$, and we then examined the distributions of the peaks in different exonic regions at each enrichment threshold. As shown in Figure 3, the detected $\mathrm{m}^{6} \mathrm{~A}$ sites with more than 8 times of enrichment are overly present near the stop codon but highly deficient near transcription starting site (TSS) and on $5^{\prime}$ UTR. The different distribution patterns of the highly and weakly methylated sites may indicate function versatility and call for more specialized analysis targeting the sites with different methylation level. In contrast to distribution preference of $\mathrm{m}^{6} \mathrm{~A}$ sites on mRNA, it is almost uniformly (or randomly) distributed on lncRNA regardless of the "IP/input ratio" specified.

In the aforementioned example, we implemented the default.

3.2. Case Study 2: RNA 5-Methylcytosine from BS-Seq. In previous study, we confirmed that RNA ${ }^{6} \mathrm{~A}$ methylation sites are enriched near stop codon and discovered that highly enriched $\mathrm{m}^{6} \mathrm{~A}$ methylation sites are depleted on $5^{\prime} \mathrm{UTR}$. Next, we study 5 -methylcytosine $\left(\mathrm{m}^{5} \mathrm{C}\right)[20,21]$ profiled with RNA BS-Seq experiment $[11,22]$. Different from the relatively wellstudied DNA ${ }^{5} \mathrm{C}$ methylation $[23,24]$, the functions of RNA $\mathrm{m}^{5} \mathrm{C}$ on mRNA are still largely elusive [20], and its global distributions on mRNA and lncRNA are poorly characterized so far.

The bisulfite sequencing data characterizing the RNA $\mathrm{m}^{5} \mathrm{C}$ methylation profiles in mouse embryo fibroblast [25] was directly obtained from GEO (GSE44359) and then processed with trim_galore [26] to remove low-quality reads and adaptor sequences; the processed reads are then aligned to mouse mm10 genome assembly with Bismark [27] and 424627 Cytosine (C) residuals are called with at least 5 reads aligned. To analyze the distribution of methylated $\mathrm{C}$ residuals, we further divided all the reported $\mathrm{C}$ residuals into 3 groups based on their methylation level with a binomial test using the average methylation probability $(1.65 \%)$ of the entire transcriptome at confidence level 0.05.

It can be seen from Figure 4 that the distribution of $\mathrm{m}^{5} \mathrm{C}$ on both lncRNA and mRNA has a very strong $5^{\prime}$ bias; that is, more reported $\mathrm{C}$ residuals from BS-Seq data are on the $5^{\prime}$ UTR region. The distribution patterns of 3 groups of residuals with different methylation level are quite different 


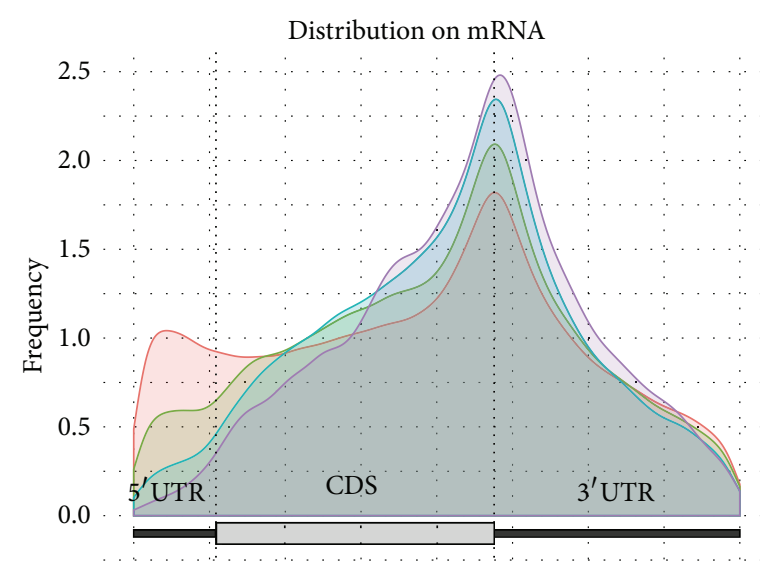

Fold enrichment

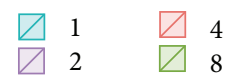

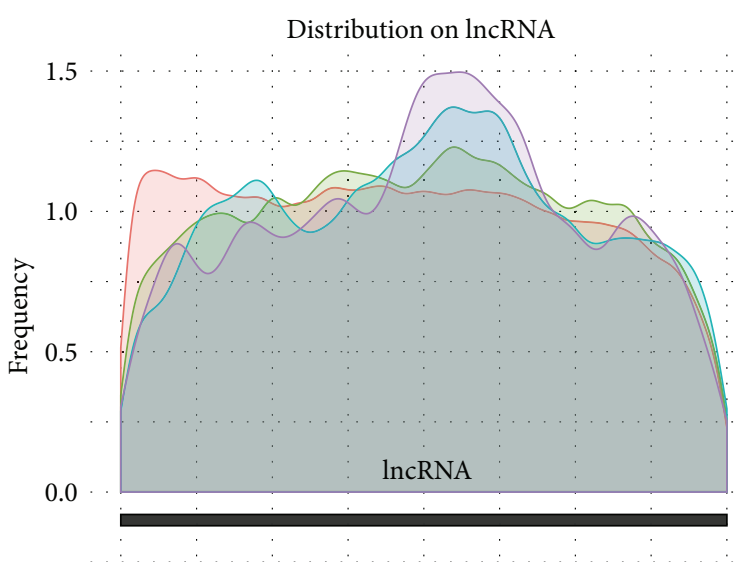

Fold enrichment

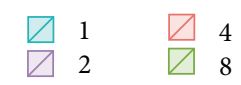

FIGURE 3: $\mathrm{m}^{6} \mathrm{~A}$ sites on mRNA and lncRNA. In mRNA, the strongest binding sites ("IP/input ratio" larger than 8) are highly enriched near stop codon side of $3^{\prime}$ UTR and deficient on TSS (transcription starting site) side of $5^{\prime}$ UTR and the phenomena are more prominent than lowly methylated sites. In contrast, the $\mathrm{m}^{6} \mathrm{~A}$ sites are almost uniformly distributed on lncRNA despite the "IP/input ratio" specified. Please note that, in this figure, the size of $5^{\prime} \mathrm{UTR}, \mathrm{CDS}$, and $3^{\prime} \mathrm{UTR}$ reflects their true width within the transcriptome, so the $5^{\prime} \mathrm{UTR}$ region is much shorter compared with the other two components. This result is based on peaks called on human HepG2 dataset [10].

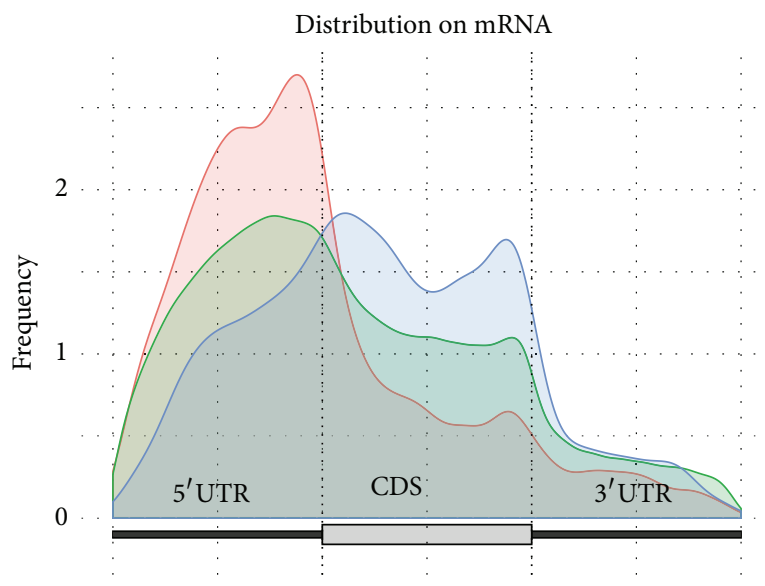

Methylation level
$\square$ Higher
$\square$ Unsure
$\square$ Lower

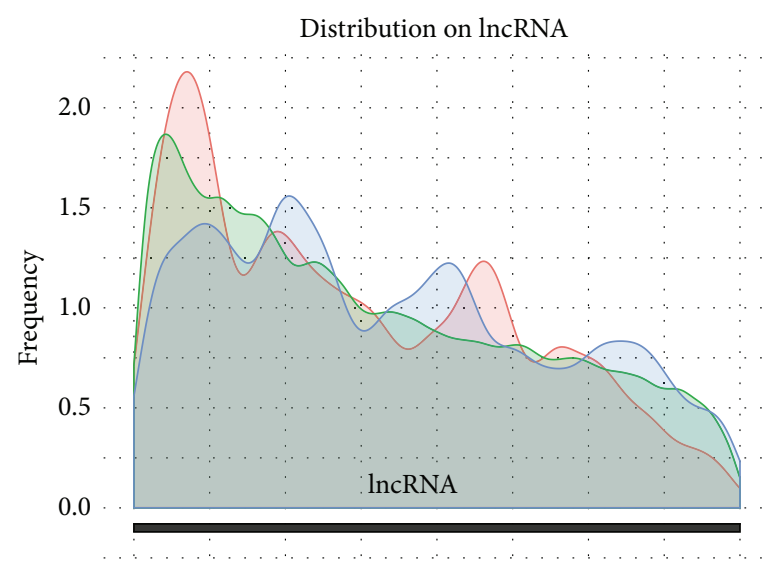

Methylation level

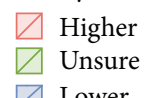

$\square$ Unsure

$\triangle$ Lower

FIGURE 4: Distribution of RNA $\mathrm{m}^{5} \mathrm{C}$ residuals on mRNA and lncRNA. We divided all the Bismark reported $\mathrm{C}$ residuals into 3 groups; that is, 9514 residuals with methylation level higher than 1.65\%, 8600 lower, and 406513 residuals cannot be determined with statistical significance. We can see that, for all 3 groups, there exists a strong $5^{\prime}$ bias on the mouse RNA BS-Seq data on both mRNA and lncRNA. The highly $\mathrm{m}^{5} \mathrm{C}$ methylated residuals are strongly enriched at $5^{\prime} \mathrm{UTR}$ of mRNA.

on mRNA, with highly methylated Cs (higher than $1.65 \%$ with 0.05 confidence level) prominently enriched on $5^{\prime} \mathrm{UTR}$ and near start codon. A cytosine residual located on $5^{\prime} \mathrm{UTR}$ is around 1 times more likely to be methylated than that on CDS and 0.68 times more likely than that on $3^{\prime} U T R$. While previous study indicates RNA $\mathrm{m}^{5} \mathrm{C}$ is enriched on both $5^{\prime} \mathrm{UTR}$ and $3^{\prime} \mathrm{UTR}$, our analysis with increased resolution indicates the enrichment is a lot stronger on $5^{\prime}$ UTR than $3^{\prime}$ UTR [20]. We also notice the pattern is complementary to the distribution of $\mathrm{m}^{6} \mathrm{~A}$, which is enriched near stop codon and $3^{\prime}$ UTR [8] (see Figure 3). These observations suggest that RNA $\mathrm{m}^{5} \mathrm{C}$ and $\mathrm{m}^{6} \mathrm{~A}$ may work in a complementary 
manner. On lncRNA, however, despite the overall $5^{\prime}$ bias, there is no apparent difference in their distribution patterns revealed.

We in the following compared the relative distribution of highly and lowly $\mathrm{m}^{5} \mathrm{C}$ methylated $\mathrm{C}$ residuals on RNA and DNA. The same processing pipe line as described previously is applied to mouse embryo fibroblast whole genome bisulfite sequencing data [25], which profiles the DNA $\mathrm{m}^{5} \mathrm{C}$ methylation rather than that on RNA. A total of 3641243 cytosine residuals are reported by Bismark, and they are then divided into 3 groups $(988335,1966017$, and 686891 residuals, resp.) based on whether their methylation level is significantly higher or lower than the average reported DNA methylation level $(65.79 \%)$ with a binomial test at 0.05 confidence level. The distributions of the 3 groups of cytosine residuals are still profiled by Guitar package with neighborhood DNA regions included, and the results are shown in Figure 5. We can see that, compared with RNA methylation profile, where the highly methylated residuals are enriched on $5^{\prime} \mathrm{UTR}$, the DNA methylation profile shows a clear oppose pattern, with the lowly methylated residuals enriched on $5^{\prime}$ UTR and peaked near the start codon to enable transcription initiation. This observation indicates DNA and RNA methyltransferase complexes probably have quite different sequence specificity, even though some key enzyme genes, such as Dnmt2 [28, 29], are shared between them [30].

\section{Discussion and Conclusion}

Currently, most biological features are represented with genome-based coordinates, making it rather tedious for comparing with transcriptomic landmarks. We developed a Guitar R package, which can be a useful tool for analyzing RNA-related genomic features, especially RNA methylation. Built upon the highly efficient GRangesList structure and GenomicFeatures R/Bioconductor packages, Guitar can efficiently process millions of genomic features within minutes for efficient transcriptomic analysis. It may automatically download gene information from UCSC genome browser, including neighborhood DNA regions, and allocate the weight of ambiguous features. The developed Guitar coordinates also provide low level conversion from transcript-based coordinates to genome-based coordinates, which should facilitate various customized analysis. Nevertheless, there are still a number of issues remaining to be addressed.

Firstly, highly abundant transcripts and ambiguous genomic features may dominate the result from Guitar package. Currently, all genomic features and all RNA transcripts have the same weight; this is fine when the genomic features are biological features such as RNA methylation sites, but it may cause problem when the genomic features are NGS mapped reads, such as RNAseq reads, because some genes are a lot more highly expressed than the other genes, and the reads generated from these genes may dominate the result. A more robust approach may be to use the median abundance of all RNA transcripts rather the summed density. It is also possible to develop a boxplot-like visualization scheme in the future to indicate the estimated higher and lower bounds for the distribution of tested features.

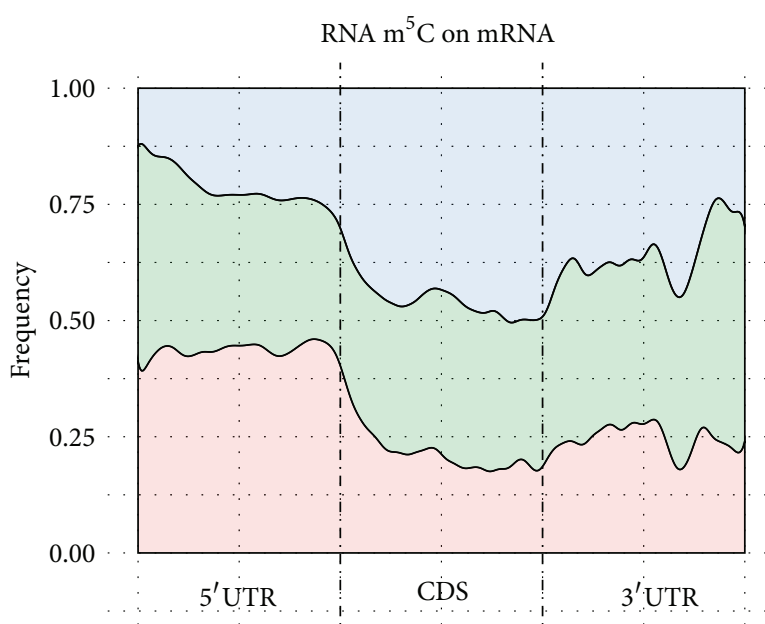

Methylation level

$\square$ Higher

$\triangle$ Unsure

$\square$ Lower

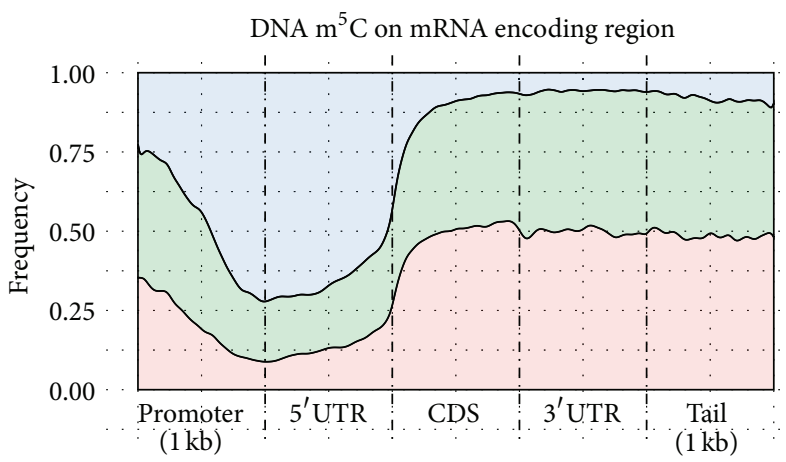

$(1 \mathrm{~kb})$

Methylation level

$\square$ Higher

$\square$ Unsure

$\square$ Lower

FIGURE 5: Distribution of DNA and RNA $\mathrm{m}^{5} \mathrm{C}$ residuals. While $C$ residuals of mRNA are likely to be methylated on $5^{\prime} \mathrm{UTR}$, it is opposite on DNA. On DNA, the start codon and $5^{\prime} \mathrm{UTR}$ regions are less likely to be methylated compared with other regions to allow the initiation of transcription process.

Secondly, the developed normalization scheme within Guitar package only facilitates the comparison of feature distribution on mRNA transcripts or on lncRNA transcripts. A cross-comparison between mRNA and lncRNA is not supported so far. In general, it is still very difficult to rigorously contrast the distribution of genomic features on mRNA and lncRNA due to their intrinsic difference. Compared with mRNA, lncRNA usually has lower expression level and less number of exons and is less conserved in sequence.

Thirdly, because of the heterogeneity of transcriptome and the discrepancy between transcriptomic and genomic features, ambiguity arises and cannot be resolved perfectly. Previous study of transcriptomic distribution of RNA methylation relies on the longest transcript to eliminate ambiguity 
in feature assignment, which may require further justification on why the longest transcript should be the canonical one. A more reasonable solution may be to rely on the most abundant isoform transcript; however, the isoform quantification is usually a difficult problem, and such information may not be handy and sometimes may be unavailable at all. Currently, Guitar package adapts two strategies to address this issue. Firstly, highly ambiguous genomic features or transcripts are excluded from the analysis. Secondly, the weight of ambiguous mapping is evenly divided among transcripts. It should be possible to develop a more rigorous formulation, for example, with fuzzy system model [31-33], to further improve the allocation of weight from ambiguous overlapping.

Fourthly, during the development process of the Guitar package, we also realized the Guitar coordinates may potentially be used for accessing the data quality of MeRIPSeq datasets. The reproducibility, uniformity, homogeneity, and the information entropy within RNA-related sequencing data (RNA-Seq, MeRIP-Seq, and BS-Seq) can be conveniently assessed with the help of Guitar coordinates; however, this application has not been explored so far and will be our future work.

Despite the aforementioned limitations, the Guitar package is capable of sketching the approximate transcriptomic distribution of millions of genomic features within minutes in a single line of command. We believe that the newly developed Guitar coordinates and the Guitar package have achieved stable performance on the data tested and should effectively facilitate the analysis of RNA methylation data and other RNA-related biological features.

\section{Additional Points}

Project name is Guitar. Project homepage is http://bioconductor.org/packages/Guitar/. Operating system(s) is Platform Independent. Programming language is R. License is GPL-2. For manual and guide, please see supplementary materials.

\section{Competing Interests}

The authors declare that they have no competing interests.

\section{Authors' Contributions}

Jia Meng, Yufei Huang, and Shao-Wu Zhang conceived the project. Xiaodong Cui, Zhen Wei, and Jia Meng carried out development and implementation of the software, drafted the paper, and carried out development and implementation of the software. Lin Zhang, Hui Liu, and Lei Sun provided valuable discussion and helped draft the paper. All authors read and approved the final paper. Xiaodong Cui and Zhen Wei contribute equally to this work.

\section{Acknowledgments}

This study is supported by National Natural Science Foundation of China (61401370, 61473232, 91430111, 61501466, and 61301220), Fundamental Research Funds for the Central Universities (2014QNB47, 2014QNA84), Jiangsu Science and Technology Program (BK20140403), and US National Institutes of Health 5 U54 CA113001 and R01GM113245. The authors also appreciate the computational support from Computational Systems Biology Core, University of Texas at San Antonio funded by National Institute on Minority Health and Health Disparities (G12MD007591) from the National Institutes of Health of USA.

\section{References}

[1] M. Lawrence, W. Huber, H. Pagès et al., "Software for computing and annotating genomic ranges," PLoS Computational Biology, vol. 9, no. 8, Article ID e1003118, 2013.

[2] N. Liu and T. Pan, "RNA epigenetics," Translational Research, vol. 165, no. 1, pp. 28-35, 2015.

[3] X. Wang, B. S. Zhao, I. A. Roundtree et al., "N(6)-methyladenosine modulates messenger RNA translation efficiency," Cell, vol. 161, no. 6, pp. 1388-1399, 2015.

[4] C. R. Alarcón, H. Lee, H. Goodarzi, N. Halberg, and S. F. Tavazoie, " $N$-methyladenosine marks primary microRNAs for processing," Nature, vol. 519, no. 7544, pp. 482-485, 2015.

[5] N. Liu, Q. Dai, G. Zheng, C. He, M. Parisien, and T. Pan, "N6methyladenosine-dependent RNA structural switches regulate RNA-protein interactions," Nature, vol. 518, no. 7540, pp. 560564, 2015.

[6] Y. Wang, Y. Li, J. I. Toth, M. D. Petroski, Z. Zhang, and J. C. Zhao, " $N{ }^{6}$-methyladenosine modification destabilizes developmental regulators in embryonic stem cells," Nature Cell Biology, vol. 16, no. 2, pp. 191-198, 2014.

[7] S. Geula, S. Moshitch-Moshkovitz, D. Dominissini et al., "m6A mRNA methylation facilitates resolution of naïve pluripotency toward differentiation," Science, vol. 347 , no. 6225 , pp. $1002-$ 1006, 2015.

[8] K. D. Meyer, Y. Saletore, P. Zumbo, O. Elemento, C. E. Mason, and S. R. Jaffrey, "Comprehensive analysis of mRNA methylation reveals enrichment in $3^{\prime}$ UTRs and near stop codons," Cell, vol. 149, no. 7, pp. 1635-1646, 2012.

[9] D. Dominissini, S. Moshitch-Moshkovitz, M. Salmon-Divon, N. Amariglio, and G. Rechavi, "Transcriptome-wide mapping of $N^{6}$-methyladenosine by $\mathrm{m}^{6} \mathrm{~A}$-seq based on immunocapturing and massively parallel sequencing," Nature Protocols, vol. 8, no. 1, pp. 176-189, 2013.

[10] D. Dominissini, S. Moshitch-Moshkovitz, S. Schwartz et al., "Topology of the human and mouse $\mathrm{m}^{6} \mathrm{~A}$ RNA methylomes revealed by $\mathrm{m}^{6}$ A-seq," Nature, vol. 484, no. 7397, pp. 201-206, 2012.

[11] M. Schaefer, T. Pollex, K. Hanna, and F. Lyko, "RNA cytosine methylation analysis by bisulfite sequencing," Nucleic Acids Research, vol. 37, no. 2, article e12, 2009.

[12] J. Meng, X. Cui, M. K. Rao, Y. Chen, and Y. Huang, "Exomebased analysis for RNA epigenome sequencing data, Bioinformatics, vol. 29, no. 12, pp. 1565-1567, 2013.

[13] J. Meng, Z. Lu, H. Liu et al., "A protocol for RNA methylation differential analysis with MeRIP-Seq data and exomePeak R/Bioconductor package," Methods, vol. 69, no. 3, pp. 274-281, 2014.

[14] H. Liu, M. A. Flores, J. Meng et al., "MeT-DB: a database of transcriptome methylation in mammalian cells," Nucleic Acids Research, vol. 43, no. 1, pp. D197-D203, 2015. 
[15] L. Liu, S.-W. Zhang, Y.-C. Zhang et al., "Decomposition of RNA methylome reveals co-methylation patterns induced by latent enzymatic regulators of the epitranscriptome," Molecular BioSystems, vol. 11, no. 1, pp. 262-274, 2015.

[16] L. Shen, N. Shao, X. Liu, and E. Nestler, "Ngs.plot: quick mining and visualization of next-generation sequencing data by integrating genomic databases," BMC Genomics, vol. 15, article 284, 2014.

[17] C. Ginestet, "ggplot2: elegant graphics for data analysis," Journal of the Royal Statistical Society: Series A (Statistics in Society), vol. 174, no. 1, pp. 245-246, 2011.

[18] Y. Fu, D. Dominissini, G. Rechavi, and C. He, "Gene expression regulation mediated through reversible $\mathrm{m}^{6} \mathrm{~A}$ RNA methylation," Nature Reviews Genetics, vol. 15, no. 5, pp. 293-306, 2014.

[19] K. D. Meyer and S. R. Jaffrey, "The dynamic epitranscriptome: N6-methyladenosine and gene expression control," Nature Reviews Molecular Cell Biology, vol. 15, no. 5, pp. 313-326, 2014.

[20] J. E. Squires, H. R. Patel, M. Nousch et al., "Widespread occurrence of 5-methylcytosine in human coding and noncoding RNA," Nucleic Acids Research, vol. 40, no. 11, pp. 50235033, 2012.

[21] S. Hussain, J. Aleksic, S. Blanco, S. Dietmann, and M. Frye, "Characterizing 5-methylcytosine in the mammalian epitranscriptome," Genome Biology, vol. 14, article 215, 2013.

[22] Y. Motorin, F. Lyko, and M. Helm, "5-Methylcytosine in RNA: detection, enzymatic formation and biological functions," Nucleic Acids Research, vol. 38, no. 5, Article ID gkpl117, pp. 1415-1430, 2009.

[23] C. Bock, "Analysing and interpreting DNA methylation data," Nature Reviews Genetics, vol. 13, no. 10, pp. 705-719, 2012.

[24] P. A. Jones, "Functions of DNA methylation: islands, start sites, gene bodies and beyond," Nature Reviews Genetics, vol. 13, no. 7, pp. 484-492, 2012.

[25] V. Khoddami and B. R. Cairns, "Identification of direct targets and modified bases of RNA cytosine methyltransferases," Nature Biotechnology, vol. 31, no. 5, pp. 458-464, 2013.

[26] F. Krueger, Trim Galore. A wrapper tool around Cutadapt and FastQC to consistently apply quality and adapter trimming to FastQ files, with some extra functionality for MspI-digested RRBS-type (Reduced Representation Bisufite-Seq) libraries, 2013.

[27] F. Krueger and S. R. Andrews, "Bismark: a flexible aligner and methylation caller for Bisulfite-Seq applications," Bioinformatics, vol. 27, no. 11, Article ID btr167, pp. 1571-1572, 2011.

[28] A. Dong, J. A. Yoder, X. Zhang, L. Zhou, T. H. Bestor, and $\mathrm{X}$. Cheng, "Structure of human DNMT2, an enigmatic DNA methyltransferase homolog that displays denaturant-resistant binding to DNA," Nucleic Acids Research, vol. 29, no. 2, pp. 439448, 2001.

[29] M. Schaefer, T. Pollex, K. Hanna et al., "RNA methylation by Dnmt2 protects transfer RNAs against stress-induced cleavage," Genes \& Development, vol. 24, no. 15, pp. 1590-1595, 2010.

[30] M. A. Machnicka, K. Milanowska, O. O. Oglou et al., "MODOMICS: a database of RNA modification pathways-2013 update," Nucleic Acids Research, vol. 41, no. 1, pp. D262-D267, 2013.

[31] C. L. P. Chen, J. Wang, C.-H. Wang, and L. Chen, "A new learning algorithm for a fully connected neuro-fuzzy inference system," IEEE Transactions on Neural Networks and Learning Systems, vol. 25, no. 10, pp. 1741-1757, 2014.
[32] J. Zhou, C. L. P. Chen, L. Chen, and H.-X. Li, "A collaborative fuzzy clustering algorithm in distributed network environments," IEEE Transactions on Fuzzy Systems, vol. 22, no. 6, pp. 1443-1456, 2014.

[33] M. Gan, C. L. P. Chen, H.-X. Li, and L. Chen, "Gradient radial basis function based varying-coefficient autoregressive model for nonlinear and nonstationary time series," IEEE Signal Processing Letters, vol. 22, no. 7, pp. 809-812, 2015. 

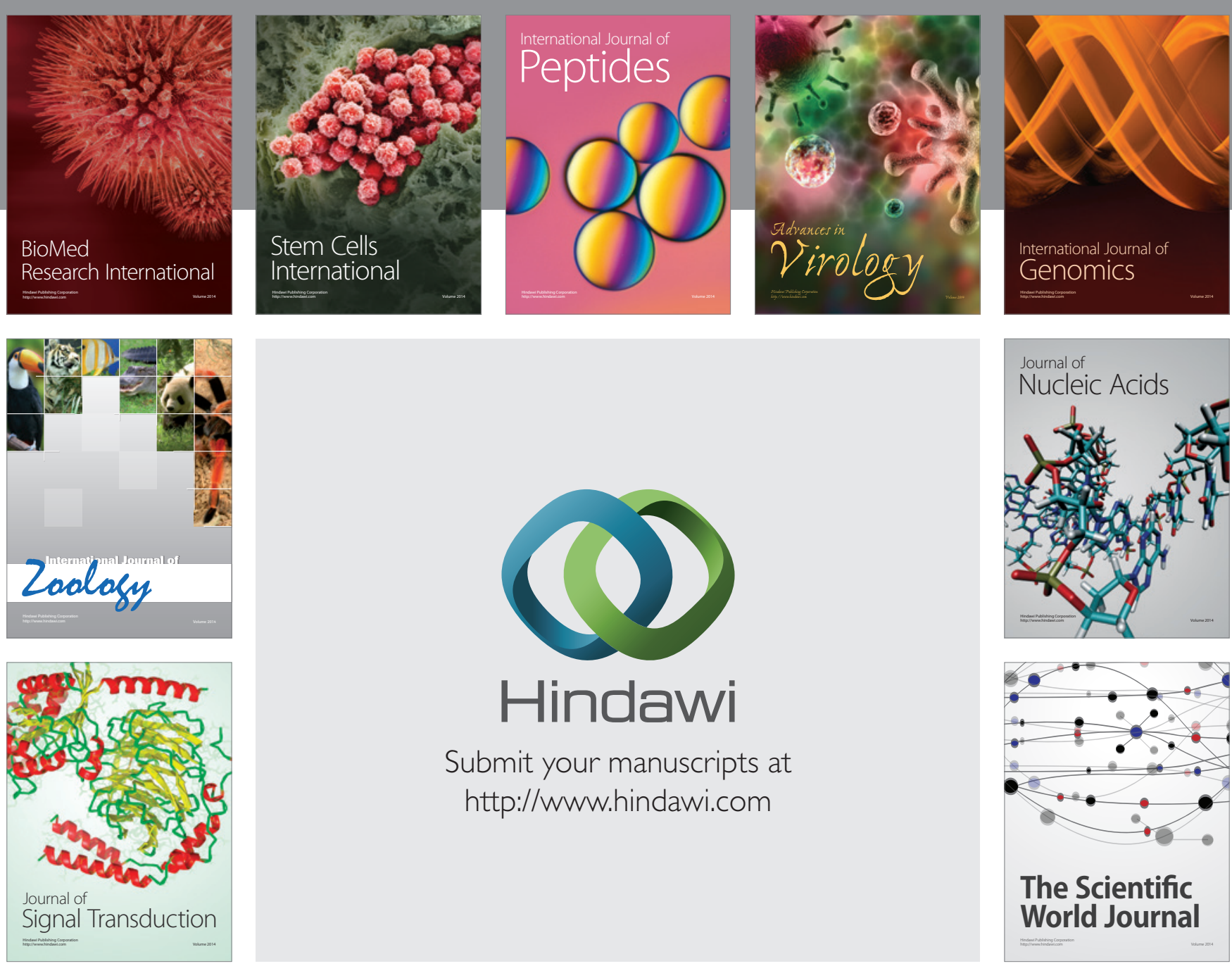

Submit your manuscripts at

http://www.hindawi.com
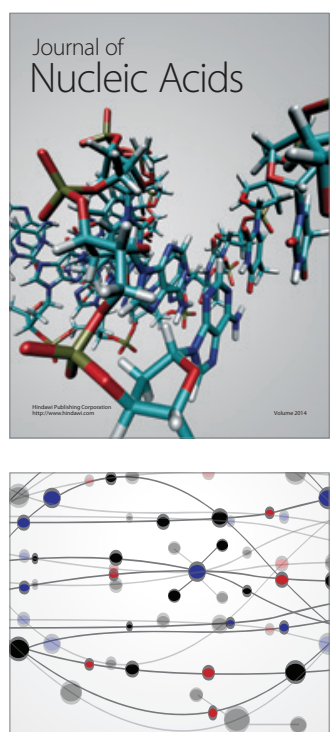

The Scientific World Journal
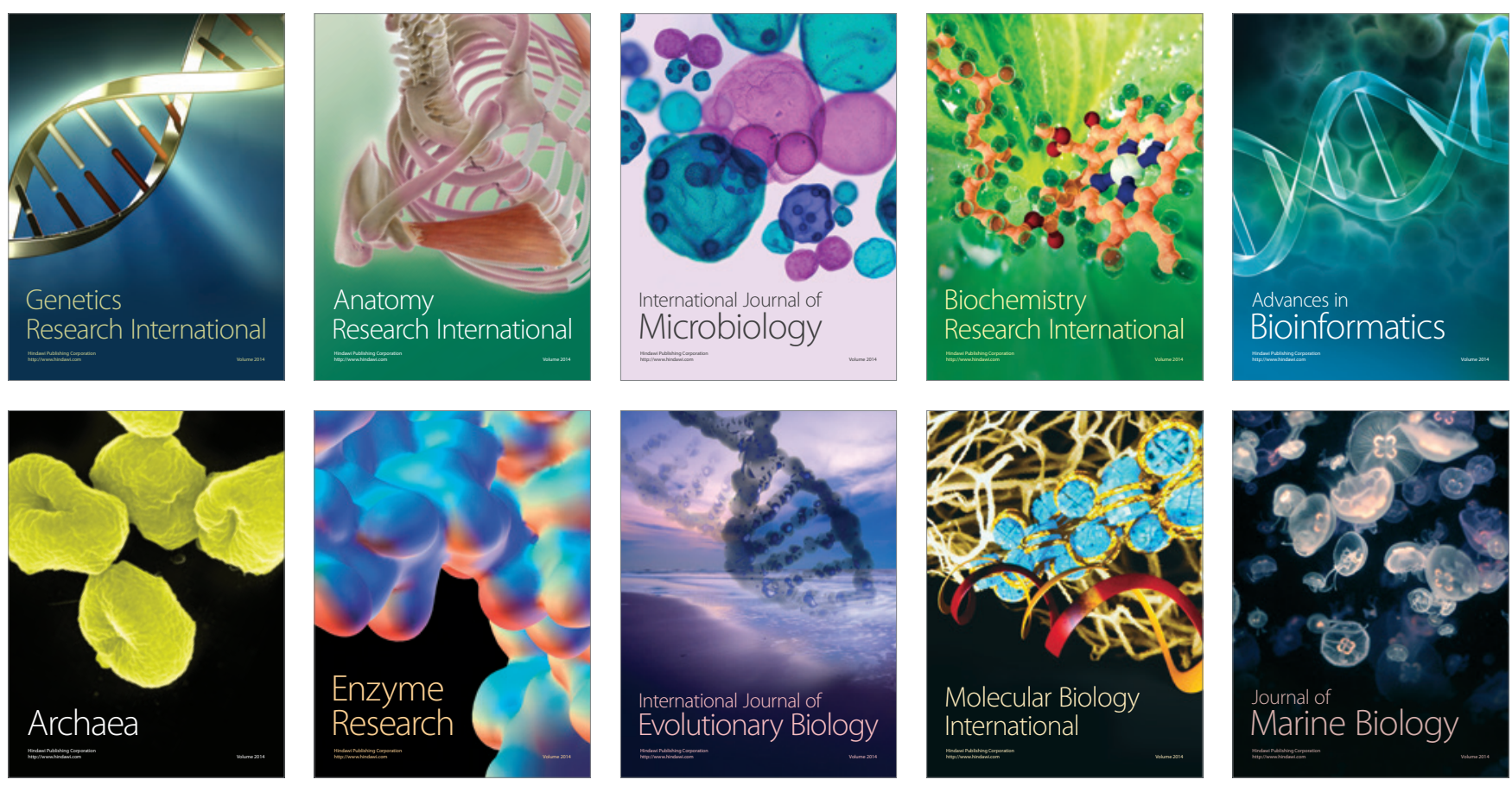